\title{
PERFORMANCE BOUNDS FOR UNEQUALLY PUNCTURED TERMINATED CONVOLUTIONAL CODES
}

\author{
Nilofer.Sk \\ Assistant professor, ECE, Mallareddy engineering college for women, AP, India
}

\begin{abstract}
The main objective of this paper is to discuss about the performance of the punctured convolution codes. Generally the punctured convolution codes are designed for a protection purpose in CDMA.In this paper a systematic method to construct performance upper bound for a punctured convolution code with an arbitrary pattern is proposed. At the decoder side perfect channel estimation is assumed and to represent the relation between input information bits and their corresponding Hamming weight outputs a weight enumerator is defined. Finally the simulations results provided very well agree with their predicted results with the upper bounds.
\end{abstract}

Keywords: Convolution code, upper bound, weight enumerator, rate matching.

\section{INTRODUCTION}

Efficiency of spectrum utilization is a very important factor in wireless communications. Several multiple access schemes are proposed to share radio spectrum which is finite and constrained. Therefore available spectrum is efficiently used by sharing the spectrum simultaneously among several individual users and this process must be done without severer degradation. And most of the wireless communications requirehigh spectral efficiency. Therefore for achieving high spectral efficiency definitely there is a need for high rate codes which maintains the system requirements such as SNR, BER and FER (frame-error rate) etc. punctured convolutional codes are high rate codes which are used to achieve high spectral efficiency [1]. Puncturing is a well known and most suitable technique to achieve high data rates. In the process of puncturing a $\mathrm{CC}$ (Convolution Code) can be extracted from mother code with less code rate. At receiver side in two subsequent separate processing steps equalization and decoding are performed [1]. Whenever the equalization is completed bit probability $1 / 2$ is inserted before performing decoding when the symbols punctured. To generate higher data rates some of the coded symbols are punctured periodically in convolutional punctured codes and the process of periodic puncturing is not an optimum method. In this project, initially based on the transfer function of the Viterbi decoded convolution code and assumption of that input sequence is infinite with equal error rates the convolutional bounds will be obtained. However center position bits contains higher error rate than the end position bits. Similarly if the frame length decreases gradually then the bound of BER becomes loose and FER (Frame Error Rate) upper bound was designed for terminated trellis code. But it is difficult to achieve upper bounds for terminated CCs using conventional transfer function.
A systematic method is proposed to obtain the performance upper bounds for a terminated CC. for obtaining performance upper bounds a weight enumerator is defined to represent the relation between input information bits and their corresponding Hamming weight outputs. A modified trellis diagram is proposed to compute the weigh enumerator [4][5].Remaining of this paper arranged as: section II clearly discusses about system model of the proposed method. Section IV consists of brief explanation about proposed bounds where as section IV consists of analysis of results. Finally in section $\mathrm{V}$ we conclude this paper.

\section{SYSTEM MODEL}

This part of the paper presents a system model for terminated CC. we assume an arbitrary puncturing. Assume a frame with $\mathrm{L}_{1}$ information bits with $\mathrm{L}_{2}$ zeros as tail bits. Therefore the input of the convolutional encoder is $\mathrm{L}_{1}+\mathrm{L}_{2}$ bits and these inputs bits are encoded at a rate of $1 / \mathrm{n}$.

Therefore after the process of convolutional encoding the number of convolutional coded bits is

$$
\mathrm{N}_{\mathrm{s}}=\mathrm{n} .\left(\mathrm{L}_{1}+\mathrm{L}_{2}\right)(1)
$$

$\mathrm{N}_{\mathrm{s}}$ is the total number of convolutional coded symbols.

For generating required length of frame and matching rate some of the coded symbols are punctured. The symbol position which is going to puncture isdefined using puncturing pattern as

$$
\mathrm{P}=\left[\mathrm{p}_{1}, \mathrm{p}_{2}, \cdots \mathrm{p}_{\mathrm{N}_{\mathrm{s}}}\right](2)
$$


$\mathrm{p}_{\mathrm{j}}=0, \mathrm{j}^{\text {th }}$ coded is symbol is punctured

If after convolutional process $\mathrm{N}_{\mathrm{p}}$ symbols are punctured then the effective code rate $r_{e}$ is defined as

$$
\mathrm{r}_{\mathrm{e}}=\frac{\mathrm{L}_{1}}{\mathrm{n} \cdot\left(\mathrm{L}_{1}+\mathrm{L}_{2}\right)-\mathrm{N}_{\mathrm{p}}}(3)
$$

In practical communications it is not possible to puncture the coded symbols periodically due to the restrictions on number of symbols. Therefore for getting a required rate matching aperiodical puncturing is required.

\section{PROPOSED UPPER BOUNDS}

As discussed previously with an arbitrary puncturing pattern of a terminated CC average BER of selected input symbol and upper bounds on FER are established in this section. To obtain the proposed bounds weight enumerator is defined. Therefore to obtain upper bounds single error event code words are considered for weight enumerator.

The weighting vector is defined as

$$
\mathrm{W}=\left[\mathrm{w}_{1}, \mathrm{w}_{2} \cdots \mathrm{w}_{\mathrm{L}_{1}}\right](4)
$$

$\mathrm{w}_{\mathrm{j}}=1$, means that $\mathrm{j}^{\text {th }}$ bit is considered for weight enumerator.

Therefore the weight enumerator of the convolutional code for a puncturing pattern $\mathrm{P}$ with weighing vector $\mathrm{W}$ is defined as

$$
\mathrm{T}_{\mathrm{w}, \mathrm{p}}(\mathrm{B}, \mathrm{D})=\sum_{\mathrm{i}} \sum_{\mathrm{j}} \mathrm{c}_{\mathrm{i}, \mathrm{j}}(\mathrm{w}, \mathrm{p}) \cdot \mathrm{B}^{\mathrm{i}} \mathrm{D}^{\mathrm{j}}(5)
$$

$\mathrm{c}_{\mathrm{i}, \mathrm{j}}(\mathrm{w}, \mathrm{p})$ : Total number of composed code words.

$\mathrm{J}$ : hamming weight of the output.

i: hamming weight of the input

The average Bit Error Rate of the bits can be obtained from the weight enumerator as

$$
P_{b}(w, p) \leq \frac{1}{|w|} \sum_{j=d_{\text {free }}}^{\infty} \sum_{i} i_{i, j}(w, p) \cdot P_{j}(6)
$$

$|\mathrm{w}|$ : w weighing vector's hamming weight.

$\mathrm{d}_{\text {free: }}$ code free distance

Therefore the upper bound on the average BER is obtained by allowing only one vector for the weighing vector in equation 6

$$
\mathrm{P}_{\mathrm{b}} \leq \frac{1}{\mathrm{~L}_{1}} \sum_{\mathrm{j}=\mathrm{d}_{\text {free }}}^{\infty} \sum_{\mathrm{i}} \mathrm{i}_{\mathrm{i}, \mathrm{j}}(\mathrm{w}, \mathrm{p}) \cdot \mathrm{P}_{\mathrm{j}}(7)
$$

Similarly upper bound on FER is obtained by using weigh enumerator with an all zero vector for $\mathrm{w}$

$$
P_{F E} \leq \sum_{j=d_{\text {free }}}^{\infty} \sum_{i} c_{i, j}(w, p) \cdot P_{j}(8)
$$

\subsection{Procedure for Weight Enumerator for Proposed}

\section{Bounds}

This method is purely based on trellis diagram of the terminated $\mathrm{CC}$ and chooses a CC encoder with the $2^{\mathrm{M}}$ number of states and the trellis depth $\mathrm{t}$ is $\mathrm{S}_{\mathrm{t}}\left(\mathrm{S}_{\mathrm{t}}=0,1,2 \ldots . .2^{\mathrm{M}}-1\right)$ and for the terminated $\mathrm{CC}$ the modified trellis diagram is as:

$>$ At trellis depth ' $t$ ', if any branch is merging into the ' 0 ' state then define another state $\mathrm{S}_{\mathrm{t}}=2^{\mathrm{M}}$ and connect every new state to a trellis depth $\mathrm{t}-1$ with a gain of 1 .

$>$ Similarly if any branch is diverging 0 states then connect 0 states from $\mathrm{t}-1$ to $\mathrm{t}$.

$>$ More than two branches are merged using an adder.

The branch gain between the $S_{t-1}$ and $S_{t}$ states is defined as $\mathrm{G}_{\mathrm{St}-1}, \mathrm{~S}_{\mathrm{t}}(\mathrm{B}, \mathrm{D})$ and it modified as per puncturing pattern as

$$
\mathrm{G}_{\mathrm{S}_{\mathrm{t}-1} \mathrm{~S}_{\mathrm{t}}}(\mathrm{B}, \mathrm{D})=\mathrm{B}^{\mathrm{x}\left(\mathrm{S}_{\mathrm{t}-1}, \mathrm{~S}_{\mathrm{t}}\right)} \mathrm{D}^{\mathrm{y}\left(\mathrm{S}_{\mathrm{t}-1}, \mathrm{~S}_{\mathrm{t}}\right)}
$$

B: input Hamming weights of $x\left(\mathrm{~S}_{\mathrm{t}-1}, \mathrm{~S}_{\mathrm{t}}\right)$

D: input Hamming weights of $y\left(S_{t-1}, S_{t}\right)$

For the state $\mathrm{S}_{\mathrm{t}} \mathrm{F}_{\mathrm{st}}(\mathrm{B}, \mathrm{D})$ is the temporary polynomial. From the trellis depth 1 the weigh enumerator is obtained by initializing $\mathrm{F}_{\mathrm{s} 0}(\mathrm{~B}, \mathrm{D})$ to 1 if $\mathrm{S}_{0}$ is 0 .

$$
\mathrm{F}_{\mathrm{S}_{\mathrm{t}}}(\mathrm{B}, \mathrm{D})=\sum_{\mathrm{S}_{\mathrm{t}-1} \in \Lambda_{\mathrm{S}_{\mathrm{t}}}} \mathrm{F}_{\mathrm{S}_{\mathrm{t}}-1}(\mathrm{~B}, \mathrm{D}) \cdot \mathrm{G}_{\mathrm{S}_{\mathrm{t}-1}, \mathrm{~S}_{\mathrm{t}}}(\mathrm{B}, \mathrm{D})_{(10)}
$$

$\Lambda_{\mathrm{S}_{\mathrm{t}}}:$ set of the states in trellis depth $\mathrm{t}-1$

Therefore now find the computation for the trellis depth $t+1$ by increasing all the trellis states at depth at $\mathrm{t}$ by 1 . And repeat this for the trellis depth $\mathrm{L}_{1}+\mathrm{L}_{2}$.

Repeat this iteration until the trellis depth $L 1+L 2$. After the completion of iteration the required weight enumerator is

$$
\mathrm{F}_{\mathrm{S}_{\mathrm{L}_{1}}+\mathrm{L}_{2}}=2^{\mathrm{M}}(\mathrm{B}, \mathrm{D})
$$


Here the proposed modified trellis diagram method consists the of $\left(\mathrm{L}_{1}+\mathrm{L}_{2}\right) \cdot 2^{\mathrm{M}}$ complexity additions. This proposed new approach is applicable to almost all practical terminated CCs decoded with a Viterbi decoder.

For understanding the proposed approach consider an modified trellis diagram for a rate $1 / 2 \mathrm{CC} \mathrm{C}_{1}$ with $\mathrm{L}_{1}=5$ and $\mathrm{L}_{2}=2$ as shown in figure 1 and the generation matrix of $\mathrm{C}_{1}$ is $\left[x^{2}+1, x^{2}+x+1\right]$

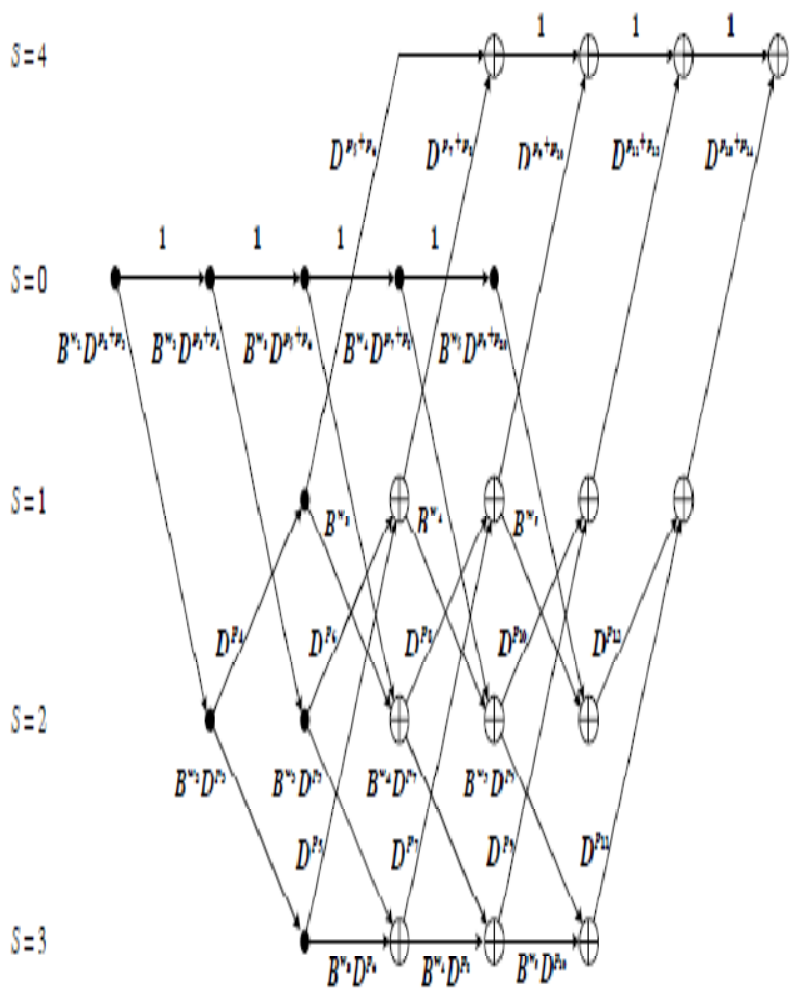

$t=0$

Fig 1: Modified trellis diagram for convolutional code $C 1$.

For obtaining the upper bound on average BER assume that all the puncturing and weighing vectors are set to 1 which means that coded symbols are not punctured and the weight enumerator is obtained as

$$
T_{1,1}(B, D)=5 B D^{5}+7 B^{2} D^{6}+8 B^{3} D^{7}+5 B^{4} D^{8}+B^{5} D^{9}(11)
$$

By using above equation (11)the upper bound on average BER is

$$
\mathrm{P}_{\mathrm{b}} \leq \mathrm{P}_{5}+2.8 \mathrm{P}_{6}+4.8 \mathrm{P}_{7}+4 \mathrm{P}_{8}+\mathrm{P}_{9}(12)
$$

The transfer function for the $\mathrm{CC}_{1}$ is computed as

$$
\mathrm{T}(\mathrm{B}, \mathrm{D})=\mathrm{BD}^{5} /(1-2 \mathrm{BD})=\mathrm{B}^{1} \mathrm{D}^{5}+2 \mathrm{~B}^{2} \mathrm{D}^{6}+4 \mathrm{~B}^{3} \mathrm{D}^{7}+\cdots
$$

The conventional upper bound on BER is computed for this code as

$$
\mathrm{P}_{\mathrm{b}} \leq \mathrm{P}_{5}+4 \mathrm{P}_{6}+12 \mathrm{P}_{7}+32 \mathrm{P}_{8}+\cdots
$$

Therefore the upper bound for considered example and simulation results are shown in figure 2 . And it is clear that the proposed bound is tighter than conventional bound. Thereforer the by setting the corresponding $\mathrm{i}^{\text {th }}$ component $\mathrm{w}_{\mathrm{i}}$ of weighing vector, upper bound for the $i^{\text {th }}$ information bit is calculated as $\mathrm{w}_{\mathrm{i}}=1$ for $\mathrm{j}=\mathrm{i}$, oterwise 0

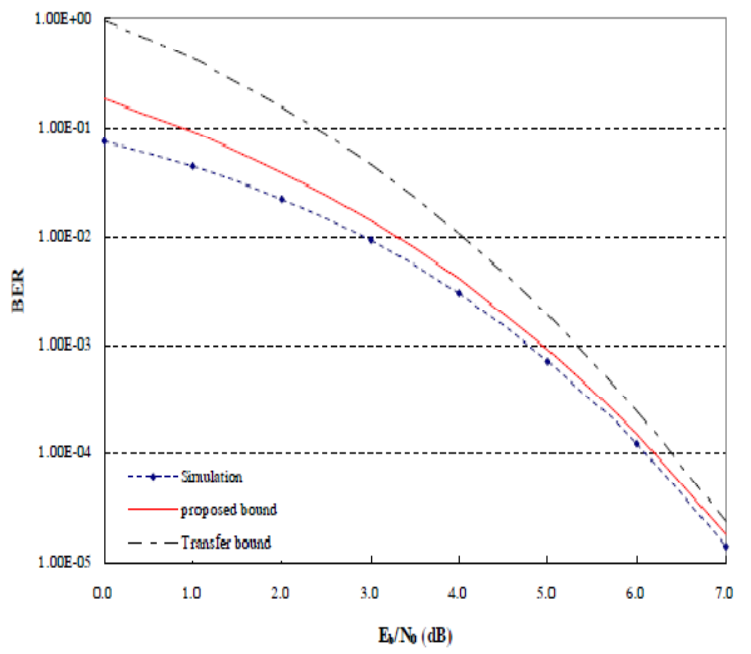

Fig 2: BER performance of convolutional code $C 1$.

For the terminated $\mathrm{CC} \mathrm{C}_{2}$, for the purpose of rate matching the weight enumerator is obtained before the puncturing and it is as

$$
\begin{aligned}
& T_{1,1}(B, D)=29 B D^{18}+54 B^{2} D^{18}+53 B^{3} D^{18}+99 B^{4} D^{20} \\
& +42 B^{5} D^{20}+21 B^{6} D^{20}+\cdots
\end{aligned}
$$

Due to the puncturing the performance of the CC degraded. With the end position symbols are punctured with the puncturing pattern $\mathrm{p}^{2}$. The weight enumerator of the $\mathrm{CC}$ after the puncturing is defined as 


$$
\begin{aligned}
& T_{1, p^{2}}(B, D)=2 B^{2} D^{10}+B^{3} D^{10}+5 D^{11}+3 B^{2} D^{11} \\
& +4 B^{3} D^{11}+B^{4} D^{11}+3 B^{5} D^{11}+B^{6} D^{11}+B^{7} D^{11} \\
& +3 B^{1} D^{12}+2 B^{2} D^{12}+5 B^{3} D^{12}+2 B^{4} D^{12}+B^{5} D^{12} \\
& +4 B^{6} D^{12}+2 B^{7} D^{12}+\cdots
\end{aligned}
$$

Therefore by comparing equation 15 and 16 it is clear that distance is decreased from 18 to 10 with the puncturing. Therefore the upper bound on FER and average BER are as

$$
\begin{aligned}
& \mathrm{P}_{\mathrm{FE}} \leq 3 \mathrm{P}_{10}+14 \mathrm{P}_{11}+22 \mathrm{P}_{12}+\cdots \\
& \mathrm{P}_{\mathrm{b}} \leq \frac{1}{29}\left(7 \mathrm{P}_{10}+51 \mathrm{P}_{11}+99 \mathrm{P}_{12}+\cdots\right)
\end{aligned}
$$

Therefore by applying the proposed method on $\mathrm{CC} \mathrm{C}_{2}$, the performance results are shown in Fig 3-Fig 6. Here $C_{2}$ is terminated convolutional code in the second part of HS-SCCS. And the coded output Hamming distances are less than dmin +10 , where $\mathrm{d}_{\min }$ is the minimum distance between the codewords.

\section{RESULTS}

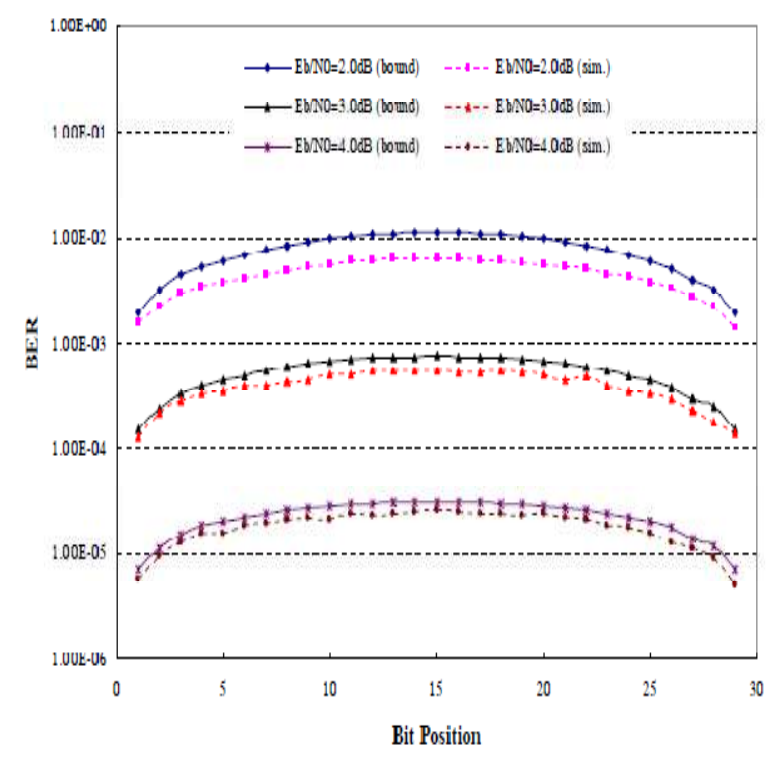

Fig.3: BER performance of $C 2$ for different bit positions (before end puncturing).

Above figure represents the simulation results for $\mathrm{CC} \mathrm{C}_{2}$ and upper bound on BER before end puncturing. And at the center position bits the error rate is 4 times larger than those of end position bits. At $\mathrm{E}_{\mathrm{b}} / \mathrm{N}_{0}=2.0 \mathrm{~dB}$ and $4.0 \mathrm{~dB}$, the 15-th information bit error rate is 4.3 and 4.0 times larger than that of the first information bit, respectively

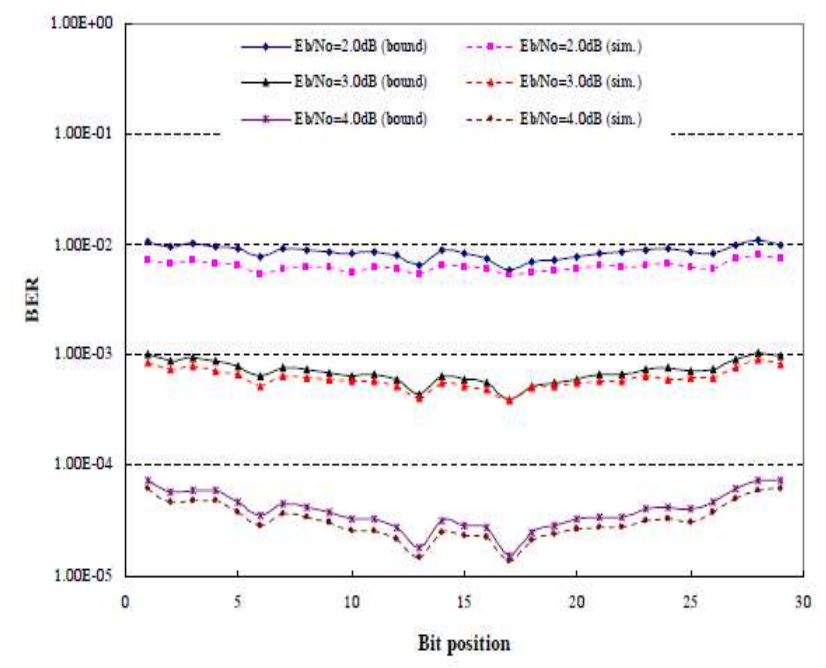

Fig. 4:BER performance of $C 2$ for different bit positions (after end puncturing).

Similarly fig 4 represents the simulation results of $\mathrm{CC} \mathrm{C}_{2}$ and upper bound on BER after end puncturing. From the results it is clear that depending on the position of the bit in the frame the variation of the BER is small with low $E_{b} / N_{0}$. Since the minimum distance of the end position bits are decreased compared to center position bits the BER of the center position bits is low.

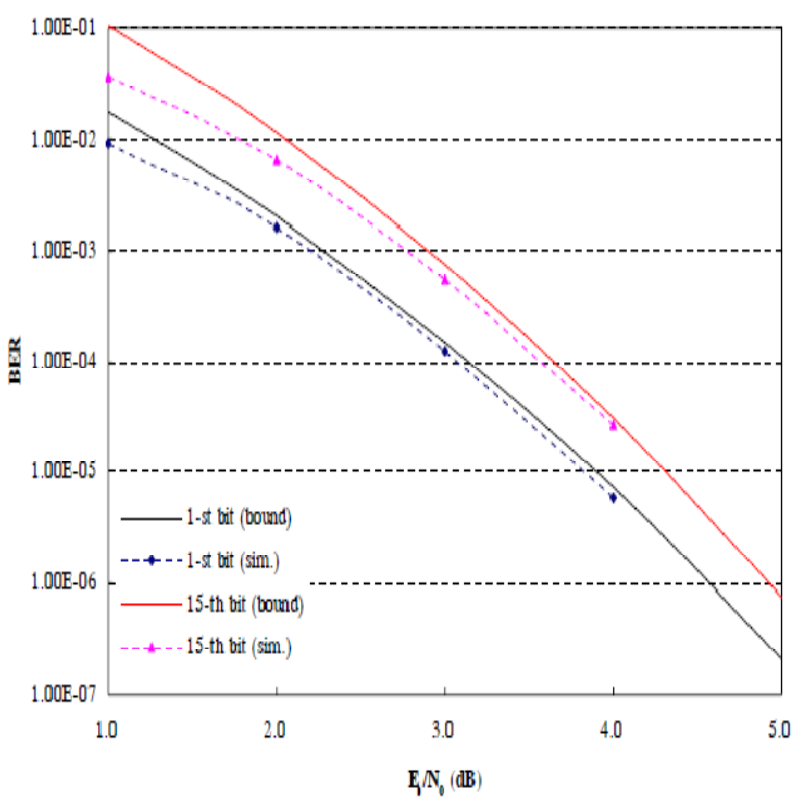

Fig 5:BER performance of $C 2$ before end puncturing. 
Figure 5 shows simulation results for the initial and $15^{\text {th }}$ information bits in $\mathrm{C}_{2}$ before the puncturing. The performance difference present between initial and $15^{\text {th }}$ bit is $0.6 \mathrm{~dB}$. And with the increment in $\mathrm{E}_{\mathrm{b}} / \mathrm{N}_{0}$ the performance difference is maintained regularly.

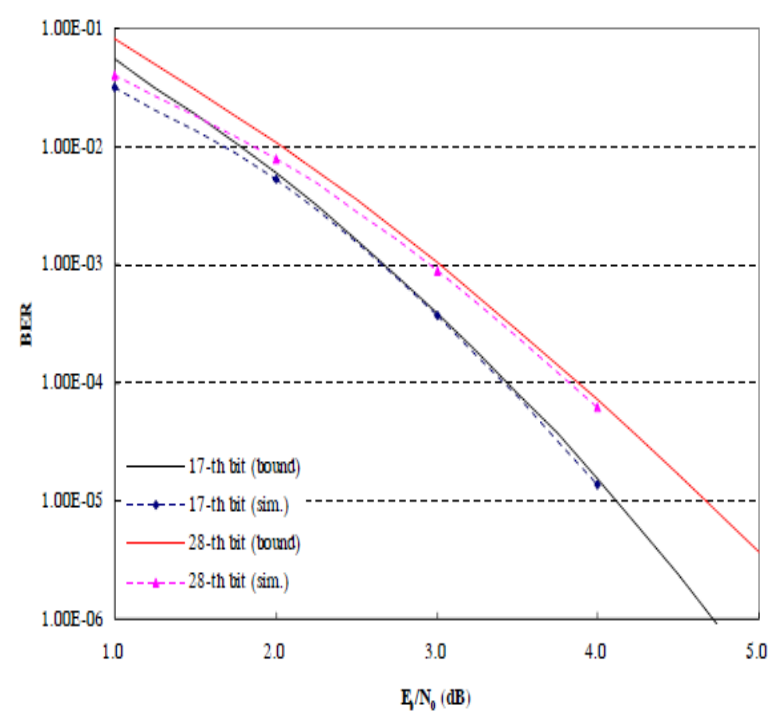

Fig.6:BER performance of $C 2$ after end puncturing.

Similarly figure 6 represents simulation results for the information bits of $17^{\text {th }}$ and $28^{\text {th }}$ positions. Here the $17^{\text {th }}$ bit shows the best BER performance where as $28^{\text {th }}$ bit shows the worst BER performance. At low $\mathrm{E}_{\mathrm{b}} / \mathrm{N}_{0}$ both the bits are similar to each other. When compared to $17^{\text {th }}$ bit, the $28^{\text {th }}$ bit performance increases more slowly with increment in $\mathrm{E}_{\mathrm{b}} / \mathrm{N}_{0}$.

\section{CONCLUSIONS}

Using this project performance of upper bounds for a terminated CC with arbitrary puncturing is proposed and weight enumerator is proposed to compute proposed bounds which define the relation between the input information bit and their corresponding Hamming code outputs. Using the coefficients of weight enumerator the upper bounds on average BER and on FER are computed. Here the proposed method is based on modified trellis diagram by considering the puncturing pattern and finally the results show that the proposed bounds are tighter to the actual performance of terminated CCs.

\section{REFERENCES}

[1]. A. J. Viterbi, "Convolutional codes and their performance in communication systems," IEEE Trans. Commun. Tech., vol. COM-19, no. 5, 751-772, Oct. 1971.

[2]. R. J. McEliece, The Theory of Information and Coding - A Mathematical Framework for Communication. Boston, MA: Addison-Wesley, 1977.
[3]. C. Berrou, A. Glavieux, and P. Thitimasjshima, "Near Shannon limit error-correcting coding and decoding: turbo codes," in Proc. IEEE International Conf. Commun., 1993, pp. 1064-1070.

[4]. C. Berrou, "The ten-year-old turbo codes are entering into service,"IEEE Commun. Mag., vol. 41, no. 8, pp. 110-116, Aug. 2003.

[5]. 3GPP, "Multiplexing and channel coding (FDD)," 3GPP TS25.212, v. 6.8.0, June 2006.

[6]. J. Cain, G. Clard, and J. Geister, "Punctured convolutional codes ofrate $(n-1) / n$ and simplified maximum likelihood decoding," IEEETrans. Inform Theory, vol. 25, no. 1, pp. 97100, Jan. 1979.

[7]. J. Hagenauer, "Rate compatible punctured convolutional codes andtheir applications," IEEE Trans. Commun., vol. 36, no. 4, pp. 389-400,Apr. 1988

[8]. Y. Yasuda, K. Kashiki, and Y. Hirata, "High rate punctured convolutional codes for soft decision viterbi decoding," IEEE Trans. Commun., vol. 32, no. 3, pp. 315-319, Mar. 1984.

[9]. G. Caire and E. Viterbo, "Upper bound on the frame error probability of terminated trellis codes," IEEE Commun.Lett., vol. 2, no. 1, pp. 2-4, Jan. 1998.

[10]. K. M. Rege and S. Nanda, "Irreducible FER for convolutional codes with random bit puncturing: application to CDMA forward channel," in Proc. IEEE Veh. Technol. Conf., 1996, pp. 1336-1340.

[11]. H. Moon, "Improved upper bound on bit error rate for truncatedconvolutional codes," IEE Electron. Lett., vol. 34, no. 1, pp. 65-66,Jan. 1998

[12]. H. Moon and D. C. Cox, "Improved performance upper bound forterminated convolutional codes," IEEE Commun. Lett., vol. 11, no. 6,pp. 519-521, June 2007.

[13]. H. Moon and D. C. Cox, "Performance upper bounds for terminated convolutional codes," in Proc. IEEE Wireless Commun.Networking Conf., 2003, pp. 252-256.

\section{BIOGRAPHIES}

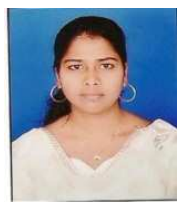

Ms .Shaik. Nilofer is working as an Assistant Professor in Mallareddy engineering College for women, Hyderabad. She has 3 yearsteaching experience at UG level. 